\title{
Working memory as persistent neural activity
}

Joshua J. Foster, Edward K. Vogel, and Edward Awh

Department of Psychology and Institute for Mind and Biology, University of Chicago

In Press. To appear in: M.J. Kahana and A.D. Wagner (Eds.), Oxford Handbook of Human Memory. Oxford University Press.

\begin{abstract}
Working memory (WM) is an online memory system that allows us to hold information "in mind" in service of ongoing cognitive processing. Here, we emphasize that short-term retention of information typically involves an interplay between WM and long-term memory (LTM), especially when task demands or interruptions divert our focus from remembered items. We suggest that active neural representation may distinguish between "online" representations in WM and "offline" representations in LTM. This perspective is at odds with "activity-silent" models of WM, which hold that WM representations can be sustained without persistent neural activity. We suggest that activity-silent representations might be more productively conceptualized as offline representations in LTM because accessing these representations shows multiple signatures of retrieval from LTM. Moreover, active neural traces track WM load, predict individual differences in performance, and respect sharp item limits in WM storage. Thus, we argue that using neural activity as an operational definition of WM may provide strong traction for studying the dynamic collaboration between WM and LTM that is critical for intelligent behavior.
\end{abstract}




\section{Working memory and long-term memory are distinct systems that work together}

Working memory (WM) is a mental workspace that allows us to hold information "in mind" in service of complex cognitive tasks (Baddeley, 2003; Baddeley \& Hitch, 1974; Cowan, 2001; Luck \& Vogel, 2013). Despite strict capacity limits for WM storage (Adam, Vogel, \& Awh, 2017; Cowan, 2001; Luck \& Vogel, 1997; Zhang \& Luck, 2008), this memory system is indispensable for intelligent thought: information stored in WM can be quickly accessed, and can be flexibly updated, overwritten, and manipulated (Baddeley \& Hitch, 1974). WM is distinguished from long-term memory (LTM), which supports offline storage of vast amounts of information (Atkinson \& Shiffrin, 1968; Waugh \& Norman, 1965). Although terminology has varied ${ }^{1}$, this distinction between online and offline memory systems is at the heart of many influential models of memory (Atkinson \& Shiffrin, 1968; Cowan, 2001; LaRocque, Lewis-Peacock, \& Postle, 2014; Oberauer \& Hein, 2012; Unsworth \& Engle, 2007). In line with this perspective, one goal of this chapter is to consider how these distinct systems work together to guide ongoing cognition.

Thus far, as is standard in the literature, we have defined WM on the basis of its functional properties such as rapid accessibility. One shortcoming of this approach, however, is that almost precisely the same list of functional properties can often be attributed to LTM (Awh \& Vogel, In Press). For example, when primed by the right context, representations in LTM can be rapidly accessed and updated (Ericsson \& Kintsch, 1995). Indeed, the overlap between the functional profiles of WM and LTM have led some to question whether the distinction between WM and LTM is warranted (Crowder, 1982; Davelaar, Yonatan Goshen-Gottstein, Ashkenazi, Haarmann, \& Usher, 2005; Oztekin, Davachi, \& McEIree, 2010). However, there are several reasons to maintain the theoretical distinction between WM and LTM. First, classic neuropsychological studies have reported a double dissociation between performance on shortterm memory and long-term memory tasks. For example, studies of H.M., a patient who had undergone a bilateral medial temporal resection, revealed that he was virtually unable to form new LTM traces, despite relatively preserved performance on memory tasks with short delay periods (Scoville \& Milner, 1957). Conversely, Warrington and Shallice (1969) reported a patient with damage in perisylvian cortex who was severely impaired in phonological WM tasks, but had relatively preserved performance on classic tests of LTM. This pattern of evidence points to non-overlapping neural substrates that are critical for WM and LTM performance (but see Ranganath \& Blumenfeld, 2005 for a critique of this dissociation). Second, individual difference studies have found that WM and LTM are best modelled as separate latent variables that account for distinct variance in overall cognitive ability (Unsworth, Brewer, \& Spillers, 2009; Unsworth, Fukuda, Awh, \& Vogel, 2014; Unsworth, Spillers, \& Brewer, 2010; Unsworth et al., this volume), inconsistent with the hypothesis that a single memory system drives WM and LTM performance. Third, while LTM is strongly susceptible to proactive interference (Underwood, 1957; for review, see Anderson \& Neely, 1996), the content of WM appears to resist proactive interference (Oberauer, Awh, \& Sutterer, 2017), consistent with the hypothesis that online representations in WM have a distinct functional profile from representations in LTM. Finally,

\footnotetext{
${ }^{1}$ What we refer to as WM - the online component of memory (which we will argue is neurally active) has gone by several names other names, including primary memory (James, 1890; Waugh \& Norman, 1965), the short-term store (Atkinson \& Shiffrin, 1968), and the focus of attention (Cowan, 2001; McElree, 2006). What we refer to as LTM - the offline component of memory - is often called secondary memory (James, 1890; Unsworth \& Engle, 2007).
} 
storage in WM has been shown to generate stimulus-specific and load-specific neural activity that tracks the voluntary storage goals of the observer (Harrison \& Tong, 2009; Luck \& Vogel, 2013; Serences, Ester, Vogel, \& Awh, 2009; Sreenivasan \& D’Esposito, 2019; Vogel \& Machizawa, 2004), whereas LTM is thought to be underpinned by changes in synaptic weights (for review, see Lamprecht \& Ledoux, 2004). Thus, multiple converging lines of evidence motivate a taxonomy of memory that recognizes the neural and functional distinctions between WM and LTM.

Although there is reason to maintain a distinction between WM and LTM, most complex tasks even those that only require maintenance of information over seconds - require close collaboration between these systems. This collaboration is captured by embedded process models of memory, which conceptualize memory as a hierarchy of memory states (e.g. Cowan, 2001; McElree, 2006; Oberauer, 2002). For instance, Cowan (2001), proposed that memories exist in one of three states. First, all remembered experiences are stored offline in LTM. Second, a subset of those LTM representation, due to recency or priming, can exist in a state of heightened accessibility that has been referred to as activated LTM (although this term was never intended to imply that these representations are maintained via neurally active traces). Finally, a small subset of 3-4 items can be held in an online state, which Cowan called the focus of attention. Here, we equate WM storage with maintenance in the focus of attention, and we argue that persistent neural activity may provide a productive way to track the frequent transitions between WM and LTM that are required for most cognitive tasks.

\section{Neural activity as a means of distinguishing between working memory and long-term memory}

Embedded process models highlight the importance of studying the collaboration between WM and LTM. However, one challenge for this effort is that behavioral measures alone often do not reveal whether performance was guided by WM or LTM. To take the simplest example, information retrieved after a short delay period could have either been maintained online throughout the memory period, or it could have been offloaded and then retrieved from activated LTM (Jeneson \& Squire, 2012; McCabe, 2008; Rhodes \& Cowan, 2018; Unsworth \& Engle, 2007). Considering this challenge, we argue that neurally active representations of the memorandum may provide strong traction for distinguishing between online representations in WM and offline representations in LTM. That said, there is ongoing debate regarding the fundamental question of whether WM storage is associated with persistent neural activity (e.g. Constantinidis et al., 2018; Lundqvist, Herman, \& Miller, 2018; Miller, Lundqvist, \& Bastos, 2018; Mongillo, Barak, \& Tsodyks, 2008; Stokes, 2015). In the following sections, we discuss the evidence for "activity-silent" conceptions of WM storage, and we argue that persistent neural activity may provide a more productive demarcation between WM and LTM. In turn, we argue that this operational definition of WM may prove useful for elucidating the dynamic transitions between WM and LTM that are critical for ongoing cognition (e.g. Carlisle, Arita, Pardo, \& Woodman, 2011; Fukuda \& Vogel, 2019; Fukuda \& Woodman, 2017; Reinhart, McClenahan, \& Woodman, 2016). 


\section{Neural activity tracks the content of working memory}

The first motivation for an operational definition of WM storage based on neural activity is the longstanding evidence that persistent neural activity is tied to the content and number of representations that are voluntarily held in this online memory system. Studies in humans and non-human primates alike have shown that storage in WM is accompanied by content-specific neural activity that codes for the representations held in WM (for comprehensive reviews, see Sreenivasan, Curtis, \& D'Esposito, 2014; Sreenivasan \& D'Esposito, 2019). Initial evidence for content-specific activity came from studies in non-human primates, which showed that the delay-period neural activity varied systematically with the specific feature values stored in WM (e.g. Funahashi, Bruce, \& Goldman-Rakic, 1989; Funahashi, Chafee, \& Goldman-Rakic, 1993; Fuster \& Jervey, 1981). Multivariate pattern analysis of functional neuroimaging data has revealed similar findings in humans. Early studies showed that visual features stored in WM such as color or orientation - can be decoded from the pattern of neural activity measured with fMRI in early visual cortex (Ester, Anderson, Serences, \& Awh, 2013; Harrison \& Tong, 2009; Serences et al., 2009). For instance, Ester et al. (2013) showed that delay-period activity in early visual cortex precisely tracked the remembered orientation (Figure 1). More recent work, however, suggests that content-specific activity is broadly distributed across much of cortex, including frontal, parietal, and sensory areas (Ester, Sprague, \& Serences, 2015; Ester, Sutterer, Serences, \& Awh, 2016; Sprague, Ester, \& Serences, 2014; Yu \& Shim, 2017; for review, see Christophel, Klink, Spitzer, Roelfsema, \& Haynes, 2017). Studies that have examined content-specific activity as a function of memory load have found that multiple items can be decoded from delay-period activity, but that the fidelity of content-specific activity declines as memory load increases (Buschman, Siegel, Roy, \& Miller, 2011; Emrich, Riggall, Larocque, \& Postle, 2013; Sprague et al., 2014; Sprague, Ester, \& Serences, 2016; Sutterer, Foster, Adam, Vogel, \& Awh, 2019). Thus, the fidelity of active neural representations declines with increasing WM load in a fashion that mirrors the declines in behavioral performance that have been documented in many past studies. 
A

Working Memory Task

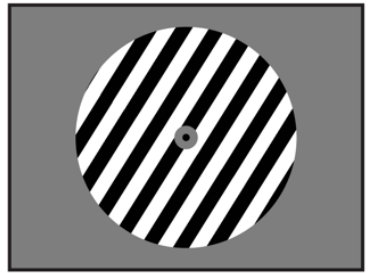

Sample (1 second)

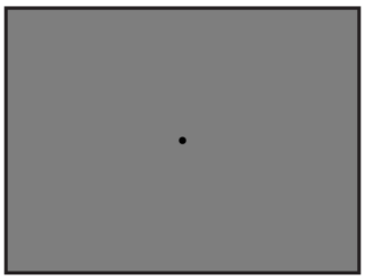

Delay (12 seconds)

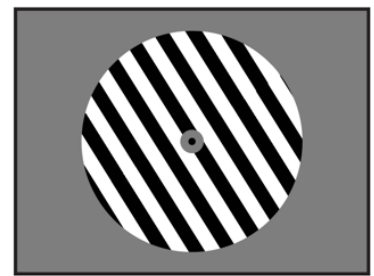

Test (3 seconds)

B

Delay-period channel-response function

(8-12 seconds after sample onset)

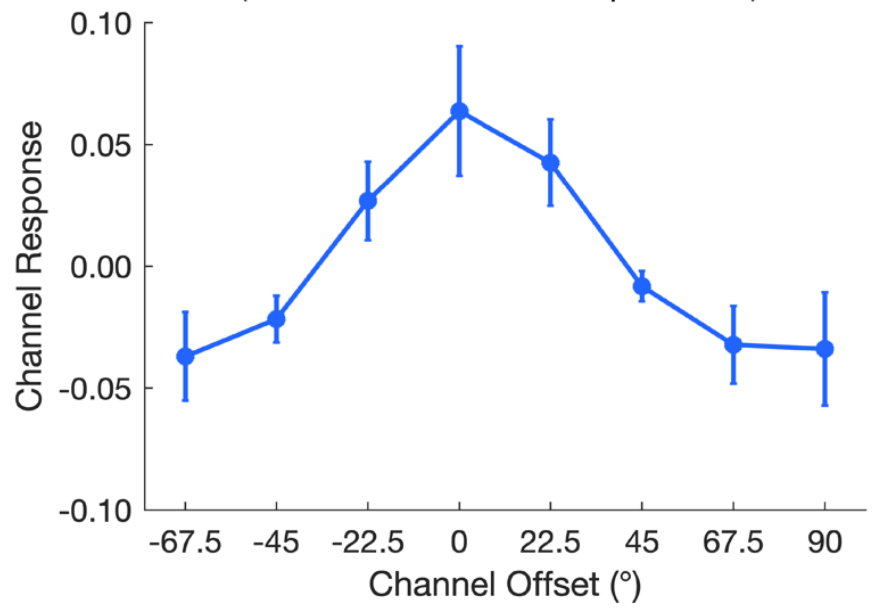

Figure 1. Content-specific neural activity that tracks features held in WM. (A) Ester and colleagues (2013) asked observers to remember and the orientation of a sample grating for a 12-second delay period, before adjusting a test grating to match the remembered orientation. (B) Using an inverted encoding model (Brouwer \& Heeger, 2009, 2011), Ester et al. (2013) reconstructed an orientation-selective channel response function from delay-period activity measure with fMRI in early visual areas (V1 and V2v), showing that delay activity in visual cortex tracked the remembered orientation. Adapted from Ester et al. (2013).

\section{Load-dependent neural activity tracks working memory load and predicts working memory capacity}

Both electroencephalograpy (EEG) and functional Magnetic Resonance Imaging (fMRI) studies have identified active neural signals that track WM load and - critically - WM performance (Fukuda, Mance, et al., 2015; Todd \& Marois, 2004, 2005; Vogel \& Machizawa, 2004). These neural signals scale with the number of items in WM, but reach an asymptote around 3-4 items, corresponding to the putative capacity of WM (Adam et al., 2017; Zhang \& Luck, 2008). For instance, Vogel and Machizawa (2004) reported a contralateral slow-wave measured with EEG that tracks the number of visual items stored in WM. In a typical task, subjects are presented with a bilateral display with the same number of objects in each hemifield, and are cued to remember the items on one side of the display (Figure 2a). Vogel and Machizawa (2004) 
measures activity at posterior electrodes contralateral and ipsilateral to the remembered stimuli. They found a sustained contralateral negativity that persisted throughout the delay period (Figure 2b), which they called Contralateral Delay Activity (CDA). Furthermore, CDA amplitude scaled with memory load and reached an asymptote at around 3-4 items (Figure 2c) (also see Fukuda, Woodman, \& Vogel, 2015). Other work has reported similar load-dependent profile in the blood-oxygen-level-dependent (BOLD) response measured in posterior parietal cortex (Todd \& Marois, 2004), and in desynchronization of posterior alpha-band power (Fukuda, Mance, et al., 2015). Critically, load-dependent signals predict individual differences in WM capacity (Fukuda, Mance, et al., 2015; Unsworth, Fukuda, Awh, \& Vogel, 2015; Vogel \& Machizawa, 2004; for a meta-analysis, see Luria, Balaban, Awh, \& Vogel, 2016), suggesting that these signals play a functional role in WM storage. In sum, active neural signals respect the voluntary storage goals of the observer, track the number of items stored, and predict the observer's success in guiding behavior with those representations. This broad array of evidence motivates our proposal to use these signals to demark the boundaries between WM and passive modes of storage.
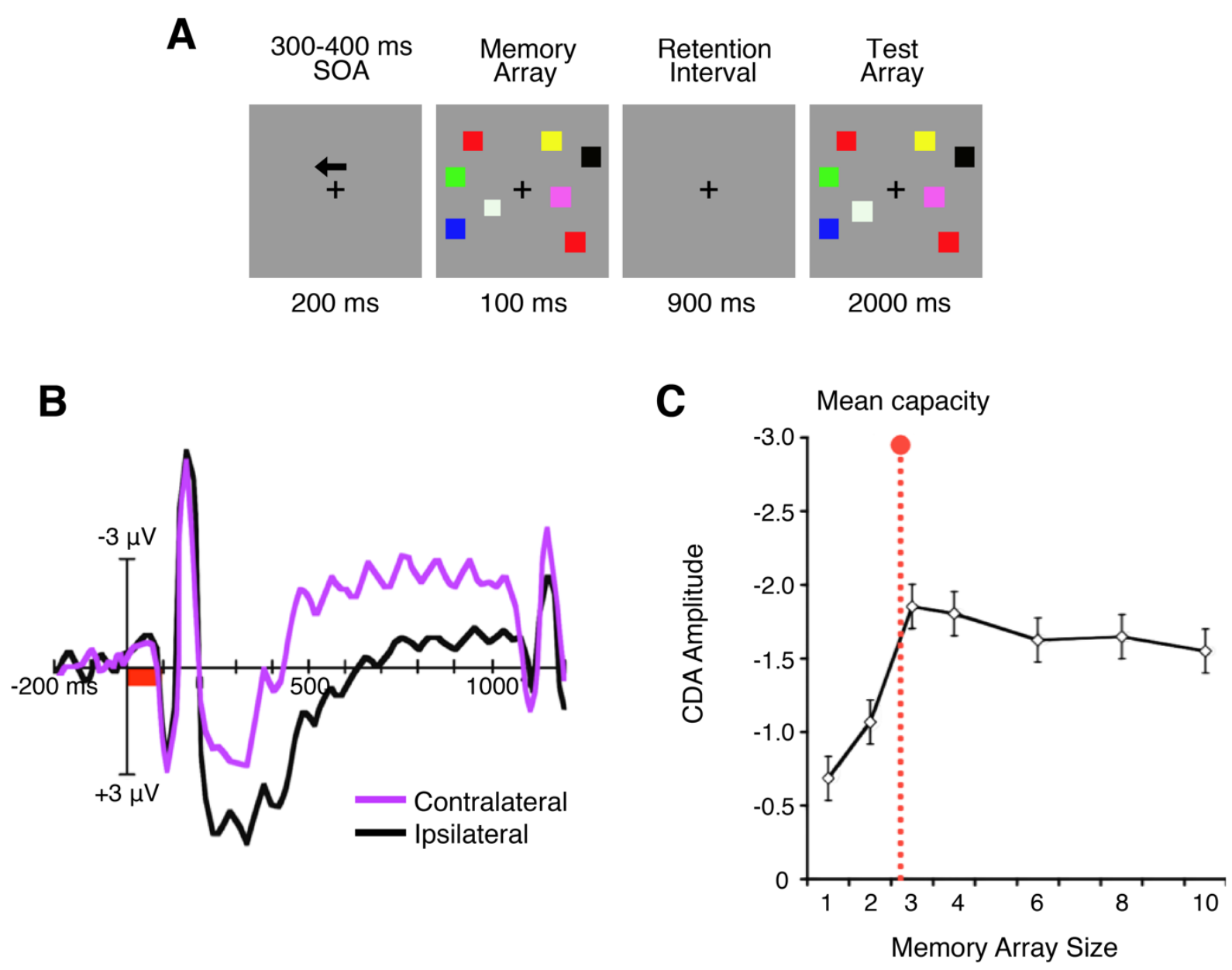

Figure 2. Delay-period activity - here Contralateral Delay Activity (CDA) - tracks memory load. (A) In a lateralized change detection task the observer must remember the items on the cued side of the memory array, and detect whether an item changed color in the test array. (C) Contralateral and ipsilateral waves 
locked to onset of the memory array at posterior electrodes. The CDA is the sustained difference between the contralateral and ipsilateral waves throughout the delay period. (C) CDA amplitude scales with the number of items to be remembered, reaching an asymptote around 3 items. The dotted line shows the mean visual working memory capacity for the sample estimated from performance in an independent change detection task.

\section{Unattended memory items are not represented by neural activity}

As we foreshadowed above, recent work has raised an important challenge to the role of persistent neural activity in WM storage. Proponents of "activity-silent" models of WM have argued that WM storage may instead be accomplished via weight-based changes in synaptic connectivity that do not require sustained neural activity (e.g. Lundqvist, Herman, \& Miller, 2018; Miller et al., 2018; Mongillo, Barak, \& Tsodyks, 2008; Postle, 2015; Stokes, 2015; Wolff, Jochim, Akyurek, \& Stokes, 2017). According to this view, persistent neural activity is best understood as reflecting attention towards prioritized memories rather than WM storage per se (LaRocque et al., 2014; Lewis-Peacock, Drysdale, Oberauer, \& Postle, 2012; Postle, 2015; Wolff et al., 2017). Though we will offer an alternative interpretation below, this view falls in line with repeated demonstrations that information that can be remembered over a short delay despite relatively long interruptions in the persistent neural activity that tracks that information.

Perhaps the clearest demonstration of short-term retention without persistent neural activity has come from tasks in which subjects must temporarily prioritize one of several remembered items. In a seminal study, Lewis-Peacock and colleagues (2012) asked subjects to remember two sample items (Figure 3a). During the delay period, a cue indicated which item would be tested by an initial memory probe, called the "attended memory item". Critically, however, the uncued item, called the "unattended memory item", also had to be remembered because that item had an equal chance of being tested during the latter part of the trial. Lewis-Peacock and colleagues (2012) found that content-specific activity was present for the attended memory item only. Following the initial cue, Lewis-Peacock et al. (2012) found that the attended memory item could be decoded from delay-period activity, but the unattended memory item could not (Figure $3 \mathrm{~b}$ and 3c). Critically, if the unattended memory item was cued in the second phase of the task, an active neural representation of the formerly unattended item was reinstated (Figure 3c), and subjects had no problem reporting this item (also see LaRocque, Lewis-Peacock, Drysdale, Oberauer, \& Postle, 2013; Larocque, Riggall, Emrich, \& Postle, 2017; Lewis-Peacock et al., 2012; Rose et al., 2016; Sprague, Ester, \& Serences, 2016; but see Christophel, lamshchinina, Yan, Allefeld, \& Haynes, 2018). A similar pattern is also seen in short-term retention tasks in which a secondary task distracts subjects from the memory task during the delay period. Here too, the sample items are remembered even though persistent activity is disrupted during the secondary task (Kiyonaga, Dowd, \& Egner, 2017; Lebedev, Messinger, Kralik, \& Wise, 2004; van Moorselaar et al., 2018; Watanabe \& Funahashi, 2014). Together, these findings suggest that short-term retention of a stimulus does not depend on uninterrupted neural activity. 

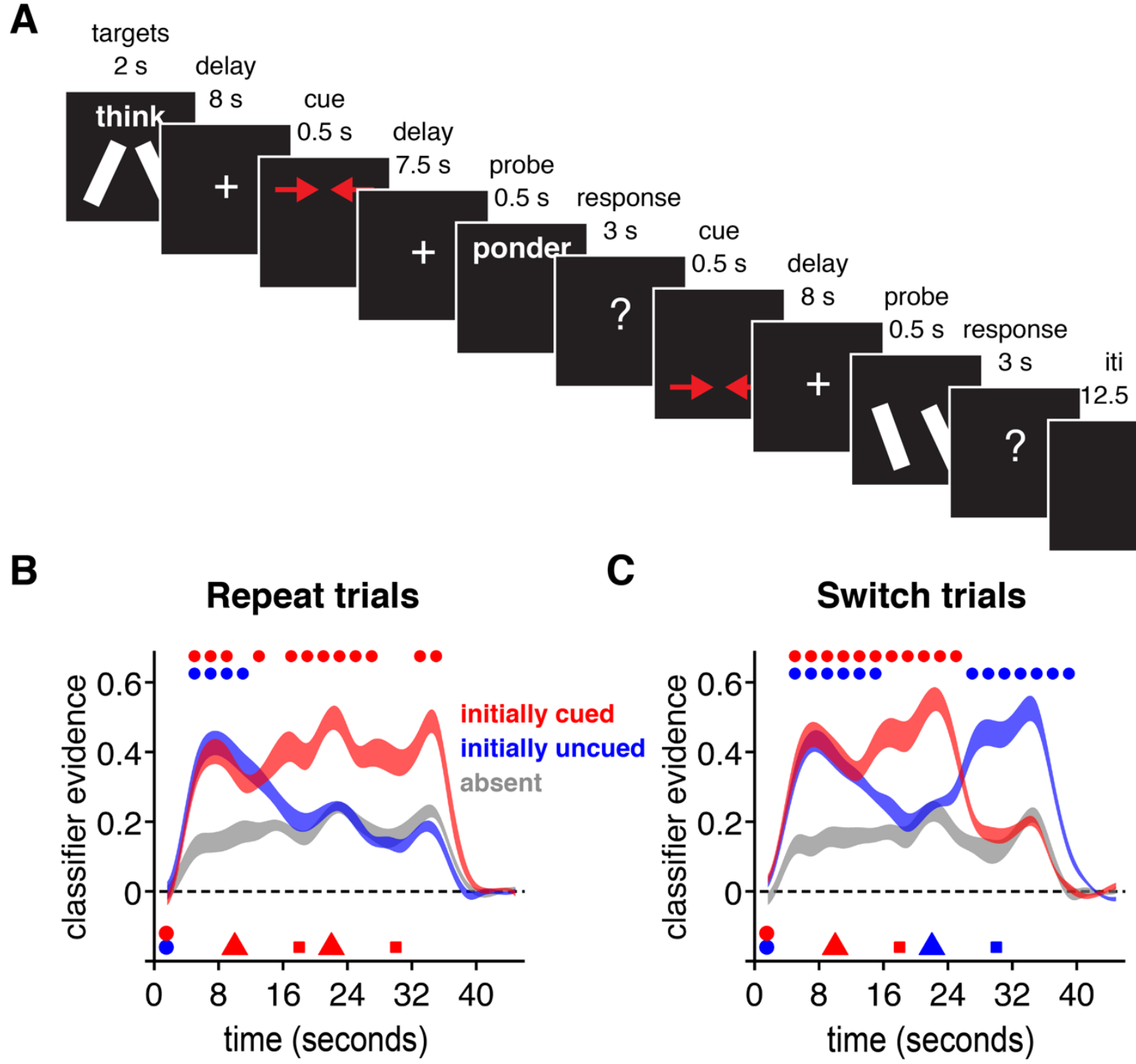

Figure 3. Unattended memory items are not represented by neural activity. (A) Lewis-Peacock et al. (2012) presented subjects with two memory items (drawn from three stimulus categories: phonological, semantic, and visual). Following a delay, a cue indicated which item would be tested by an initial recognition probe. Next, a second cue indicated which item would be tested by a second recognition probe. The plots in $(B)$ and $(C)$ show classifier evidence for the each of three stimulus categories (initially cued, initially uncued, and absent) across time based on multivariate pattern analysis of fMRI data. The absent condition in grey refers to the category that was not included in the trial, and therefore serves as an empirical baseline. (B) On repeat trials, the same item was cued during both phases of the trial. LewisPeacock et al. (2012) found that the cued item was actively represented throughout the trial, whereas the uncued item returned to baseline following the initial cue. (C) On switch trials, the item that was uncued in the first phase of the trial was cued in the second phase. Neural representation of this item was abolished following the initial cue, but was reinstated when it was cued in the second phase of the trial. Circles at the top of the plots mark times at which there reliably greater classifier evidence for the initially cued and initially uncued stimulus categories than for the absent category. At the bottom of each plot, circles mark target onset, triangles mark cue onsets, and squares mark probe onsets. Adapted from Lewis-Peacock et al. (2012). 


\section{Are unattended memory items in working memory?}

Researchers have argued that unattended memory items are stored in WM in large part because they are easily accessed after the short delay periods (on the order of seconds to tens of seconds) used in these tasks (Miller et al., 2018; Stokes, 2015; Wolff et al., 2017). This view, however, overlooks the possibility that unattended memory items are not stored in WM at all, but are instead displaced to LTM (Jeneson \& Squire, 2012; McCabe, 2008; Rhodes \& Cowan, 2018; Rose, Buchsbaum, \& Craik, 2014; Unsworth \& Engle, 2007). Thus, it is necessary to rule out the possibility that activity-silent memories are stored in LTM to justify the proposal of an activity-silent state in WM.

A second finding that has motivated a WM interpretation of these activity-silent representations is that activity-silent memories can be reactivated by non-specific input to the network encoding the memory (Rose et al., 2016; Stokes, 2015; Stokes et al., 2013; Wolff, Ding, Myers, \& Stokes, 2015; Wolff et al., 2017). Proponents of activity-silent models of WM have suggested that activity-silent working memories are maintained via temporary changes in synaptic weights that are shaped by the content of the stored information (Stokes, 2015), perhaps mediated by residual calcium at synaptic terminals (Mongillo et al., 2008). As a consequence, a non-specific input to the network will produce a different response depending on what information is held in this activity-silent format (Mongillo et al., 2008; Stokes, 2015). Recent work has supported this prediction (Rose et al., 2016; Stokes et al., 2013; Wolff et al., 2015, 2017). Rose and colleagues (2016), for instance, found that pulse of transcranial magnetic stimulation (TMS) revived a stimulus-specific pattern of EEG activity. Similarly, Wolff and colleagues (2017) found that a high-contrast visual stimulus, intended to drive a strong response in visual cortex, also transiently revived stimulus-specific activity.

An important question is whether reactivation of activity-silent memories by non-specific input can be explained by storage in LTM. At first glance, this seems unlikely because LTM retrieval typically depends on the presence of specific cues (whether external or internal) that are associated with the retrieved representation. However, much of what we know about LTM including the importance of specific retrieval cues - has come from paradigms that delay testing for minutes to months after encoding. Furthermore, in experiments using relative short delays (e.g. minutes), researchers often ask subjects to perform a distracting task, such as a crossword puzzle, during the delay (e.g. Ellis \& Hope, 1968; Glanzer \& Cunitz, 1966; Rose et al., 2014). These measures are taken to minimize the contribution of WM to memory performance. Although these strategies are well motivated, one consequence of this approach is that we know relatively little about the properties of LTM traces in the initial moments after encoding. Thus, it is arguably an empirical question whether very recently encoded LTM representations despite being offloaded from WM storage - may be highly accessible and subject to reactivation via non-specific inputs of the kind described above. As a result, more work may be needed to determine whether reactivation per se is sufficient to infer storage in WM.

A third finding that has led researchers to embrace the idea that activity-silent memories reside in WM is that reactivation by non-specific input depends on the current behavioral relevance of the representation (Rose et al., 2016; Wolff et al., 2017), a hallmark of storage in WM (Baddeley, 2003; Baddeley \& Hitch, 1974; Williams \& Woodman, 2012). For instance, Wolff and colleagues (2017) presented observers with two oriented gratings before cueing them to selectively remember one of these items. After a short-delay, once stimulus-specific activity for 
both items had dissipated, a high-contrast visual stimulus transiently reactivated the cued item but not the uncued item. Similarly, Rose and colleagues (2016) found that a TMS pulse reactivated an unattended memory item, but that the same pulse of stimulation did not reactive the unattended memory item when it was applied after a cue that indicated that the item was no longer relevant for the task. However, other studies have demonstrated reactivation of featurespecific activity even for items that were clearly irrelevant to the current task. For example, Bae and Luck (2019) used an EEG decoding approach to demonstrate reactivation of the orientation value that was stored in WM during the prior trial, despite the fact that it had already been reported, and was no longer relevant to the task. In a similar vein, serial dependence effects are seen in WM tasks such that now-irrelevant memories from past trials bias the reports on subsequent trials (Kiyonaga, Scimeca, Bliss, \& Whitney, 2017). If reactivation is not contingent on behavioral relevance, then observing reactivation does not compel the conclusion that the restored representation was stored in WM.

\section{Accessing unattended memory items reveals the fingerprints of retrieval from long-term memory}

Thus far, we have argued that the extant data may not strongly compel a WM interpretation of activity-silent memory. Another impetus for our position is that multiple lines of work, employing procedures that closely resemble those used to argue for activity-silent WM, have found that accessing unattended memory representations reveals the "fingerprints" of LTM. For example, consider the complex span paradigm (Conway et al., 2005; Daneman \& Carpenter, 1980; Turner \& Engle, 1989). In this paradigm, subjects are shown a sequence of memoranda (e.g., numbers) that they will eventually have to report. However, in between each memorandum, subjects are given a secondary task (e.g., verification of arithmetic problems) that must be completed before the next item is presented. Performance on this dual task procedure exhibits robust correlations with fluid intelligence (Conway, Kane, \& Engle, 2003), and this paradigm has provided important insights into the relationship between WM capacity and a broad range of cognitive abilities. However, while complex span tasks were intended to target WM operations (Baddeley \& Hitch, 1974; Conway et al., 2003; Daneman \& Carpenter, 1980; Turner \& Engle, 1989), there is clear evidence that LTM also plays a central role in these tasks. Unsworth and Engle $(2006,2007)$ suggested that the encoding of subsequent items in the list in combination with the cognitive demands of the secondary task (e.g. the verification of arithmetic problems) displaces memory items from capacity-limited WM, such that subjects must rely on storage of these memory items in LTM. Indeed, individual differences studies revealed that that performance on standard measures of LTM, such as free recall of word lists, predicts performance in complex span tasks, and that both LTM and WM emerge as distinct sources of variance in complex span tasks (Mogle, Lovett, Stawski, \& Sliwinski, 2008; Shipstead, Lindsey, Marshall, \& Engle, 2014; Unsworth et al., 2009, 2014, 2010; Unsworth \& Spillers, 2010). Thus, despite only brief interruptions of attention towards the memoranda in complex span tasks, analyses of individual differences suggest that performance is supported by both WM and LTM.

Complex span tasks also show other fingerprints of retrieval from LTM. It is well established that retrieving information from LTM - called retrieval practice - renders that information more accessible later on (Carrier \& Pashler, 1992; Karpicke \& Roediger, 2006; for review, see Roediger \& Butler, 2011). McCabe (2008) reasoned that if items in complex span tasks are displaced from WM and must be retrieved from LTM, then retrieving items from LTM during complex span tasks should serve as retrieval practice, improving subsequent LTM retrieval. To 
test this hypothesis, McCabe (2008) compared delayed recall for items presented in complex span tasks and simple span tasks (which do not force offloading of items from WM to LTM to the same extent as complex span tasks). In line with his hypothesis, McCabe (2008) found that delayed recall was better for items presented in a complex span task than for items presented in a simple span task (called the McCabe effect). Furthermore, McCabe found that this effect was greater for items presented earlier in the list, which afford more opportunities for retrieval from LTM throughout the trial (also see Loaiza, Duperreault, Rhodes, \& McCabe, 2014; Loaiza \& McCabe, 2012). Thus, the secondary task in complex span procedures seems to promote retrieval practice, improving subsequent memory for items encoded during complex span. ${ }^{2}$

Although there is clear evidence that LTM plays a role in complex span tasks, one might object that complex span tasks are fundamentally different than the simpler tasks in which researchers have proposed that activity-silent WM representations are involved (e.g. Lewis-Peacock et al., 2012; Stokes, 2015; Wolff, Jochim, Akyurek, \& Stokes, 2017). Unlike these simpler tasks that require subjects to remember just one or two items, complex span tasks confront subjects with memory sets that exceed the putative 3-4 item capacity of WM (Adam et al., 2017; Cowan, 2001; Luck \& Vogel, 1997; Zhang \& Luck, 2008). Thus, it stands to reason that complex span tasks may depend on LTM to a greater extent than tasks that do not exceed the capacity limit of WM. Recent work, however, has shown that supra-capacity memory loads are not a necessary condition for the recruitment of LTM in short-term retention tasks. Rose and colleagues (2014) showed that LTM supports performance in a task that required subjects to maintain only a single item over a short delay period. They varied the extent to which a second task during the delay period diverted attention from the memory item (Figure 4a). On some trials, subjects were asked to perform a math task throughout the delay period, while on other trials subjects were free to focus on the memory item. This task is a remarkably close analog of paradigms that have been shown to temporarily disrupt active neural representation of the memory item(s) (e.g. Kiyonaga, Dowd, et al., 2017; Lewis-Peacock et al., 2012). Rose and colleagues (2014) tested for two signatures of retrieval from LTM. First, they manipulated levels of processing at encoding. This manipulation allowed the authors to test whether deeper levels of processing at encoding improved immediate recall following the delay period as they do for classic LTM tasks (Craik \& Tulving, 1975). Second, following McCabe and colleagues, Rose et al. tested whether the math task (which should displace the memory item from WM) improved subsequent memory due to retrieval practice. On trials with no distracting task, immediate recall was near perfect and did not vary with level of processing at encoding. However, on trials with the distracting math task, immediate recall was worse overall, and a level-of-processing effect emerged: immediate recall was better for items encoded deeply (Figure 4b) (Rose et al., 2014; also see Rose, Craik,

\footnotetext{
${ }^{2}$ Although these findings are consistent with the idea that the secondary task in complex span procedures promotes retrieval practice, others have proposed alternative explanations. For example, Souza and Oberauer (2017) argued that better subsequent memory performance for items encoded during complex span tasks than in simple span tasks could reflect the fact that items may spend longer in WM in complex span tasks (because items are presented at a slower rate in complex span tasks than in simple span tasks). Another possibility is that items encoded into LTM during complex span task are better remembered because they are encoded as more distinct episodes since each item is separated by the secondary task (see Radvansky \& Zacks, 2017). However, as we will see below, these accounts cannot explain more recent data.
} 
\& Buchsbaum, 2015). Although ceiling levels of performance in the absence of a distracting task do not provide strong evidence against LTM retrieval in that condition, these findings provide clear positive evidence for a contribution from LTM when WM maintenance was disrupted by the math task. Moreover, a surprise free-recall task at the end of the session showed that final free recall was better for items presented in the math conditions than for items presented in the rehearse condition (Figure 4c), in line with the hypothesis that practicing retrieving those items from LTM (following offloading during the math task) enhanced subsequent LTM access. ${ }^{3}$ Thus, the broad findings suggest a greater role for LTM retrieval in the immediate recall of information following a brief interruption.

\footnotetext{
${ }^{3}$ Rose and colleagues (2014) experiment rules out the alterative accounts that have been proposed to explain the McCabe effect that we raised in the previous footnote. Rose et al.'s finding of better final free recall performance in the math conditions than in the rehearse condition cannot be explained by slower presentation (Souza \& Oberauer, 2017) of the items since all trials were a fixed length and the conditions were interleaved. It is also difficult to see how the math conditions would lead to greater contextual distinctiveness of encoding episodes since all items were encoded during clearly demarcated trials.
} 

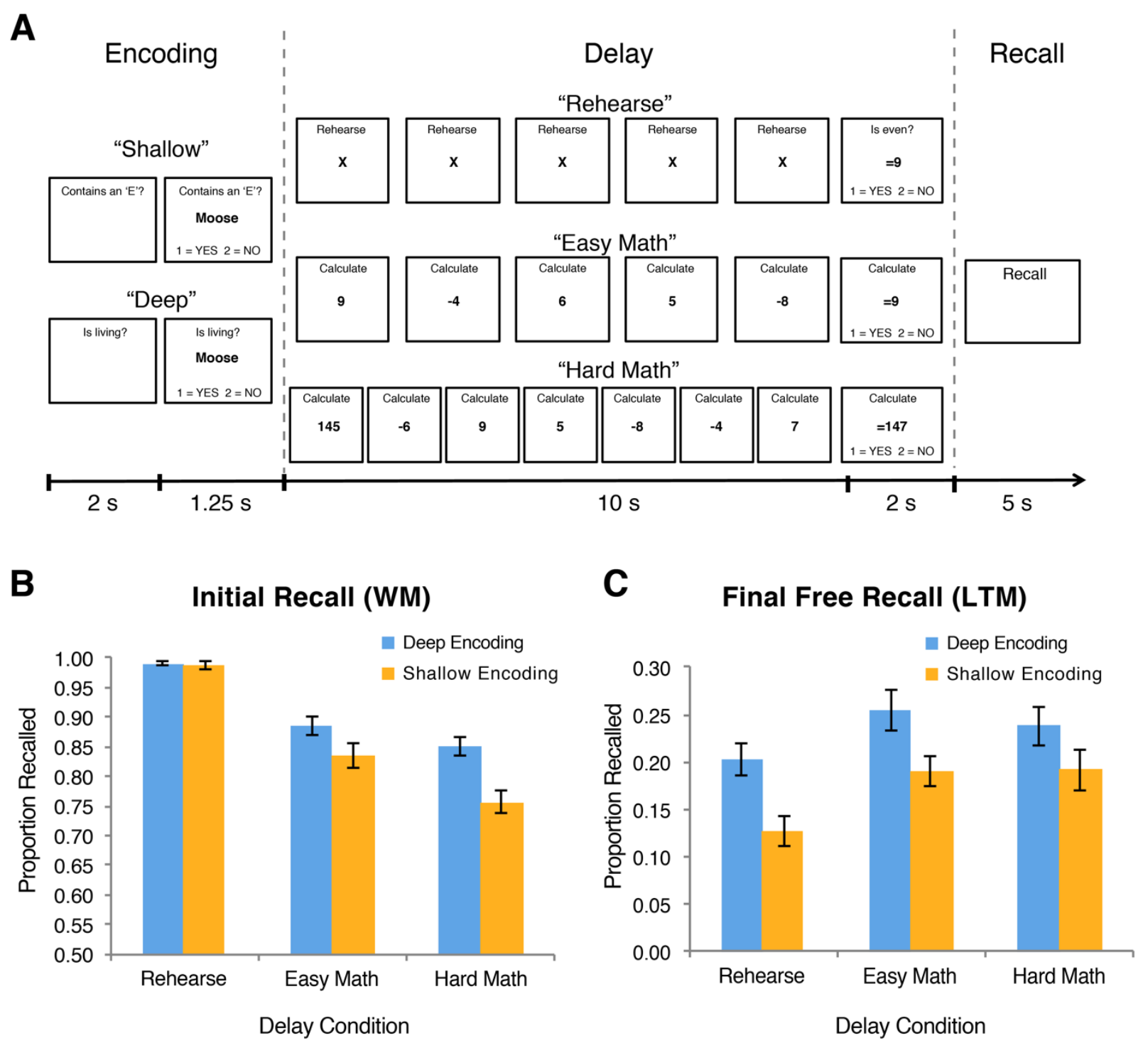

Figure 4. Accessing unattended memory items revels fingerprints of retrieval from LTM. (A) Rose and colleagues (2014) had subjects perform a WM task with two twists. First, they manipulated depth of processing at encoding. Second, they manipulated the extent to which a secondary task disrupted maintenance of the memory item. Subjects verbally reported the memory item following the delay period. (B) Recall performance immediately following the delay period. Recall performance was excellent in the rehearse condition. However, when subjects performed the distracting math task, immediate recall was worse, and a levels of processing effect emerged (i.e., better recall for item in the deep encoding condition than in the shallow encoding condition). (C) In a surprise free-recall test at the end of the session, items were better recalled in the math conditions than in the rehearse condition. This finding suggests that disruption of maintenance by the math task provided an opportunity for retrieval practice, improving free recall at the end of the session. Adapted from Rose et al. (2014). 


\section{An online-offline model of memory}

In this chapter, we have highlighted the evidence that both WM and LTM make important contributions in short-term retention tasks, with LTM playing a particularly important role when task demands force us to disengage - even briefly - from remembered information. We argue that an online-offline model of memory naturally captures these interactions between WM and LTM (Figure 5). Specifically, we suggest that as many as 3-4 representations can be stored online in WM (i.e., represented by neural activity), and that lapses in storage-related neural activity are bridged via offline storage in LTM (Jeneson \& Squire, 2012; McCabe, 2008; Rhodes \& Cowan, 2018; Rose et al., 2014; Unsworth \& Engle, 2007). Thus, through the lens of embedded process models, we propose that the boundary between WM and LTM may be best placed between the focus of attention and activated LTM, and that neurally active representations of the stimuli in the focus of attention may provide a robust means of determining whether and when a given representation is stored in this online state.

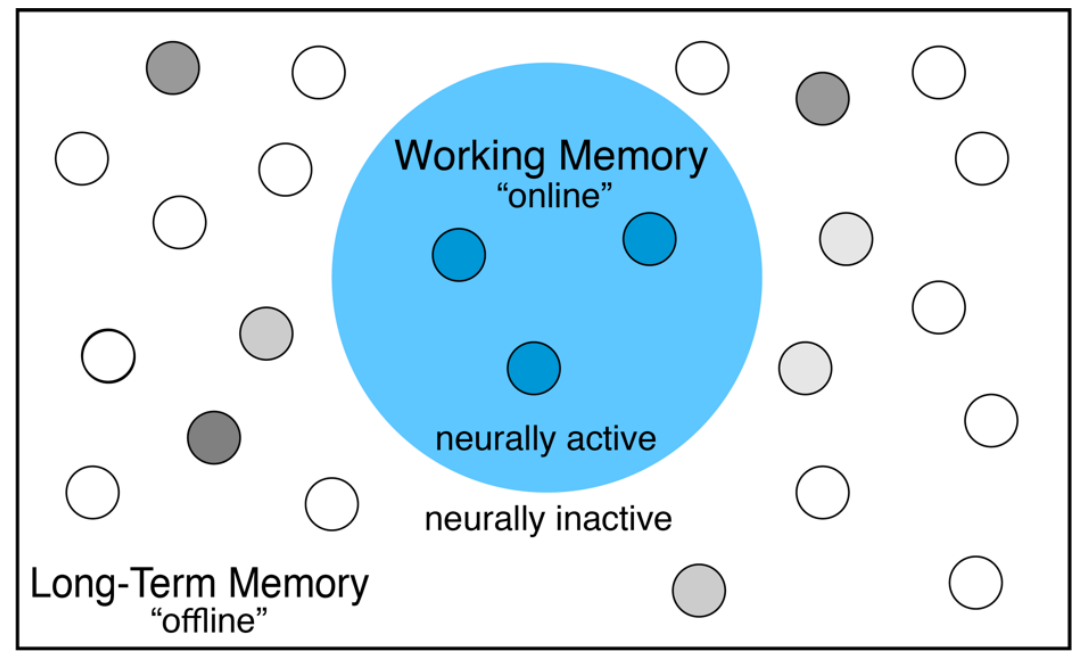

Figure 5. An online-offline model of memory. We propose that active neural representation is the dividing line WM (online memory) from LTM (offline memory). Specifically, we suggest that 3-4 representations can be stored online in WM (i.e. represented by neural activity), with all other representations passively stored in LTM (with varying levels of memory strength, represented by the shade of grey). According to this model, lapses in storage-related neural activity are bridged via passive storage in LTM.

To summarize the arguments above, there are two motivations to anchor the concept of WM to active neural signals. First, these signals capture the hallmark features of WM. WM has longbeen conceptualized as a capacity-limited system that serves as an online cognitive workspace for the storage and manipulation of information (e.g. Baddeley \& Hitch, 1974). This cognitive definition of WM almost perfectly describes what we know about active neural representations. Load-dependent neural signals reach an asymptote around memory set sizes of 3-4 (Fukuda, Mance, et al., 2015; Luria et al., 2016; Vogel \& Machizawa, 2004), in line with apparent item limits revealed by behavioral studies. Furthermore, active neural representations are under 
volitional control (Serences et al., 2009; Williams \& Woodman, 2012), and what is represented in this state can be flexibly updated depending on task demands (Christophel, Cichy, Hebart, \& Haynes, 2014; Rao, Rainer, \& Miller, 1997). Finally, active neural signals predict WM performance (Fukuda, Mance, et al., 2015; Luria et al., 2016; Todd \& Marois, 2005; Vogel \& Machizawa, 2004). Thus, multiple lines of evidence provide strong positive evidence that active neural signals index online representations in WM. Second, we have reviewed a broad array of evidence that accessing "activity-silent" representations elicits longstanding signatures of LTM access, including correlations with classic measures of LTM ability, retrieval practice effects, and levels of processing effects. Critically, these observations come from studies that employed procedures very similar to those that have been used to promote the construct of activity-silent WM. Thus, there is strong evidence for an LTM interpretation of the phenomena that have been attributed to activity-silent WM.

To the extent that WM is anchored to neural activity, an operational definition of WM storage that is based on storage-related neural activity provides a robust empirical platform for studying the dynamic interplay between WM and LTM. For example, Carlisle and colleagues (2011) set out to test theories of automaticity which propose that visual search is initially guided by an online search template in WM, but as this process is automated, the search template is offloaded to LTM. On each trial, a search array was preceded by a cue that indicated what item the subject must find in the search array. When the target item varied trial-to-trial, Carlisle and colleagues observed a robust CDA for the cue during the period between the cue and the search array, suggesting that an attentional template was actively maintained in WM. However, when the target did not change across trials, no CDA was seen for the cue, as would be expected if the attentional template had been offloaded to LTM. Finally, Carlisle and colleagues examined the cue-related CDA across repetitions of a target, and found that the amplitude of the CDA diminished across repetitions, as the template was handed off from WM to LTM. Thus, using the CDA as an active neural index of WM storage provided a productive way to track the handoff from WM to LTM.

\section{What counts as an active neural representation?}

Our proposed framework raises an important question: what counts as an active neural representation? Given that content-specific activity appears to be widely distributed across cortex (Christophel et al., 2017; Ester et al., 2015), we think that global measures of neural activity such a fMRI and EEG are more diagnostic than measurements of local populations or units, which might miss content-specific activity elsewhere. For instance, Mendoza-Halliday and colleagues found persistent content-specific spiking activity in lateral prefrontal cortex and medial superior temporal area, but not in middle temporal area (Mendoza-Halliday, Torres, \& Martinez-Trujillo, 2014). Thus, the absence of stimulus-specific activity in any given region does not preclude active representation elsewhere (also see Christophel et al., 2018). By contrast, global measures of neural activity circumvent this problem by allowing researchers to test for content-specific activity across large-scale neuronal populations. Indeed, Murray et al. (2017) recently argued that stable and sustained WM representations could be modelled from population codes despite being based on data from a large number of heterogeneous individual neurons, many of which did not exhibit sustained delay period activity. Thus, some hallmark properties of WM may emerge from large-scale network activity. 
The study by Mendoza-Halliday and colleagues (2014) raises another important consideration. Although the authors did not observe stimulus-specific spiking activity in the middle temporal area during the delay period, they did find that local field potentials (LFPs) in this region encoded the remembered feature. Indeed, this finding might explain why fMRI measures (which are sensitive to LFPs) have consistently found stimulus-specific delay activity in early sensory areas while unit recordings have not (Leavitt, Mendoza-halliday, \& Martinez-trujillo, 2017; Serences, 2016). There is now substantial evidence that field potentials encode remembered features (Foster, Sutterer, Serences, Vogel, \& Awh, 2016; Mendoza-Halliday et al., 2014; Salazar, Dotson, Bressler, \& Gray, 2012). For instance, oscillations in the alpha frequency band (8-12 Hz) encode spatial positions held in WM (Foster et al., 2016). Does content-specific oscillatory activity in the absence of spiking activity count as an active neural representation? We argue that it does. Neural oscillations are distinct from the changes in synaptic weights that are thought to encode latent memories stored in LTM. Furthermore, as Mendoza-Halliday and colleagues (2014) suggest, stimulus-specific LFPs may be the product of spiking activity elsewhere in the brain, or of spiking activity that cannot be detected by non-invasive measurements of neural activity, such as electroencephalography (EEG).

\section{Concluding remarks}

Many cognitive tasks require us to hold information "in mind" so that it can be manipulated and acted upon. Here, we have argued that embedded processes models provide a productive perspective for considering the tight collaboration between online and offline memory systems, implemented via neurally active traces and passive weight-based mechanisms. While this perspective pushes back against recent arguments for activity-silent forms of WM, we nevertheless acknowledge the importance of the phenomena that have been highlighted in this recent work. To build effective models of complex cognitive behaviors, it will be essential to engage with paradigms that encourage the dynamic transitions between online and offline states that are required for virtually any real world task. Thus, examinations of the neural signals that track the waxing and waning of neurally active memory traces (e.g. Kiyonaga, Dowd, et al., 2017; Lewis-Peacock et al., 2012; Lundqvist et al., 2018; Miller et al., 2018), and that have begun to elucidate the potential role of synaptic plasticity in temporary storage (e.g. Rose et al., 2016; Stokes, 2015; Wolff et al., 2017), are a critical step forward towards building a more complete understanding of human memory. 


\section{References}

Adam, K. C. S., Vogel, E. K., \& Awh, E. (2017). Clear evidence for item limits in visual working memory. Cognitive Psychology, 97, 79-97. https://doi.org/10.1016/j.cogpsych.2017.07.001

Anderson, M. C., \& Neely, J. H. (1996). Interference and inhibition in memory retrieval. In E. L. Bjork \& R. A. Bjork (Eds.), Memory (pp. 237-313). https://doi.org/10.1016/B978012102570-0/50010-0

Atkinson, R. C., \& Shiffrin, R. M. (1968). Human memory: A proposed system and its control processes. In Psychology of Learning and Motivation (pp. 89-195). https://doi.org/10.1016/S0079-7421(08)60422-3

Awh, E., \& Vogel, E. K. (n.d.). Online and offline memory states in the human brain. In D. Poeppel, G. R. Mangun, \& M. S. Gazzaniga (Eds.), The Cognitive Neurosciences. MIT Press.

Baddeley, A. D. (2003). Working memory: looking back and looking forward. Nature Reviews. Neuroscience, 4(10), 829-839. https://doi.org/10.1038/nrn1201

Baddeley, A. D., \& Hitch, G. (1974). Working Memory. Psychology of Learning and Motivation, 8, 47-89. https://doi.org/10.1016/b978-0-12-121050-2.50010-7

Bae, G. Y., \& Luck, S. J. (2019). Reactivation of previous experiences in a working memory task. Psychological Science, 30(4), 587-595. https://doi.org/10.1177/0956797619830398

Brouwer, G. J., \& Heeger, D. J. (2009). Decoding and reconstructing color from responses in human visual cortex. Journal of Neuroscience, 29(44), 13992-14003.

Brouwer, G. J., \& Heeger, D. J. (2011). Cross-orientation suppression in human visual cortex. Journal of Neurophysiology, 106(5), 2108-2119. https://doi.org/10.1152/jn.00540.2011

Buschman, T. J., Siegel, M., Roy, J. E., \& Miller, E. K. (2011). Neural substrates of cognitive capacity limitations. Proceedings of the National Academy of Sciences of the United States of America, 108(27), 11252-11255. https://doi.org/10.1073/pnas.1104666108

Carlisle, N. B., Arita, J. T., Pardo, D., \& Woodman, G. F. (2011). Attentional templates in visual working memory. Journal of Neuroscience, 31(25), 9315-9322.

https://doi.org/10.1523/JNEUROSCI.1097-11.2011

Carrier, M., \& Pashler, H. (1992). The influence of retrieval on retention. Memory \& Cognition, 20(6), 633-642. https://doi.org/10.3758/BF03202713

Christophel, T. B., Cichy, R. M., Hebart, M. N., \& Haynes, J. (2014). Parietal and early visual cortices encode working memory content across mental transformations. Neurolmage. https://doi.org/10.1016/j.neuroimage.2014.11.018

Christophel, T. B., Iamshchinina, P., Yan, C., Allefeld, C., \& Haynes, J.-D. (2018). Cortical specialization for attended versus unattended working memory. Nature Neuroscience, 1. https://doi.org/10.1038/s41593-018-0094-4

Christophel, T. B., Klink, P. C., Spitzer, B., Roelfsema, P. R., \& Haynes, J.-D. (2017). The distributed nature of working memory. Trends in Cognitive Sciences, 21(2), 111-124. https://doi.org/10.1016/j.tics.2016.12.007

Constantinidis, C., Funahashi, S., Lee, D., Murray, J. D., Qi, X.-L., Wang, M., \& Arnsten, A. F. T. 
(2018). Persistent spiking activity underlies working memory. Journal of Neuroscience, 38(32), 7020-7028. https://doi.org/10.1523/JNEUROSCI.2486-17.2018

Conway, A. R. A., Kane, M. J., Bunting, M. F., Hambrick, D. Z., Wilhelm, O., \& Engle, R. (2005). Working memory span tasks: A methodological review and user's guide. Psychonomic Bullentin \& Review, 12(5), 769-786. https://doi.org/10.3758/BF03196772

Conway, A. R. A., Kane, M. J., \& Engle, R. W. (2003). Working memory capacity and its relation to general intelligence. Trends in Cognitive Sciences, 7(12), 547-552. https://doi.org/10.1016/j.tics.2003.10.005

Cowan, N. (2001). The magical number 4 in short-term memory: a reconsideration of mental storage capacity. The Behavioral and Brain Sciences, 24(1), 87-114; discussion 114-85. https://doi.org/10.1017/S0140525X01003922

Craik, F. I., \& Tulving, E. (1975). Depth of processing and the retention of words in episodic memory. Journal of Experimental Psychology: General, 104(3), 268-294. https://doi.org/10.1037/0096-3445.104.3.268

Crowder, R. G. (1982). The demise of short-term memory. Acta Psychologica, 50(3), 291-323. https://doi.org/10.1016/0001-6918(82)90044-0

Daneman, M., \& Carpenter, P. A. (1980). Individual differences in working memory and reading. Journal of Verbal Learning and Verbal Behavior, 19(4), 450-466. https://doi.org/10.1016/S0022-5371(80)90312-6

Davelaar, E. J., Yonatan Goshen-Gottstein, Ashkenazi, A., Haarmann, H. J., \& Usher, M. (2005). The demise of short-term memory revisited: Empirical and computational investigations of recency effects. Psychological Review, 112(1), 3-42. https://doi.org/10.1037/0033-295X.112.1.3

Ellis, N. R., \& Hope, R. (1968). Memory processes and the serial position curve. Journal of Experimental Psychology, 77(4), 613-619. https://doi.org/10.1037/h0026064

Emrich, S. M., Riggall, A. C., Larocque, J. J., \& Postle, B. R. (2013). Distributed patterns of activity in sensory cortex reflect the precision of multiple items maintained in visual shortterm memory. Journal of Neuroscience, 33(15), 6516-6523. https://doi.org/10.1523/JNEUROSCI.5732-12.2013

Ericsson, K. A., \& Kintsch, W. (1995). Long-Term Working Memory. Psychological Review, 102(2), 211-245.

Ester, E. F., Anderson, D. E., Serences, J. T., \& Awh, E. (2013). A neural measure of precision in visual working memory. Journal of Cognitive Neuroscience, 25(5), 754-761.

Ester, E. F., Sprague, T. C., \& Serences, J. T. (2015). Parietal and frontal cortex encode stimulus-specific mnemonic representations during visual working memory. Neuron, 87, 113. https://doi.org/10.1016/j.neuron.2015.07.013

Ester, E. F., Sutterer, D. W., Serences, J. T., \& Awh, E. (2016). Feature-Selective Attentional Modulations in Human Frontoparietal Cortex. 36(31), 8188-8199. https://doi.org/10.1523/JNEUROSCI.3935-15.2016

Foster, J. J., Sutterer, D. W., Serences, J. T., Vogel, E. K., \& Awh, E. (2016). The topography of alpha-band activity tracks the content of spatial working memory. Journal of Neurophysiology, 115(1), 168-177. https://doi.org/10.1152/jn.00860.2015 
Fukuda, K., Mance, I., Vogel, E. K., Vision, V., Neuroscience, C., \& Fukuda, K. (2015). Alpha power modulation and event-related slow wave provide dissociable correlates of visual working memory . Journal of Neuroscience, 35(41), 1-17. https://doi.org/10.1523/JNEUROSCI.5003-14.2015

Fukuda, K., \& Vogel, E. K. (2019). Visual short-term memory capacity predicts the "bandwidth" of visual long-term memory encoding. Memory \& Cognition. https://doi.org/10.3758/s13421-019-00954-0

Fukuda, K., \& Woodman, G. F. (2017). Visual working memory buffers information retrieved from visual long-term memory. Proceedings of the National Academy of Sciences, (905), 1-6. https://doi.org/10.1073/pnas.1617874114

Fukuda, K., Woodman, G. F., \& Vogel, E. K. (2015). Individual differences in visual working memory capacity: Contributions of attentional control to storage. Mechanisms of Sensory Working Memory: Attention and Performance XXV, 105-119. https://doi.org/10.1016/B9780-12-801371-7.00009-0

Funahashi, S., Bruce, C. J., \& Goldman-Rakic, P. S. (1989). Mnemonic coding of visual space in the monkey's dorsolateral prefrontal cortex. Journal of Neurophysiology, 61, 331-349. https://doi.org/10.1016/j.neuron.2012.12.039

Funahashi, S., Chafee, M. V., \& Goldman-Rakic, P. S. (1993). Prefrontal neuronal activity in rhesus monkeys performing a delayed anti-saccade task. Nature, 365(6448), 753-756. https://doi.org/10.1038/365753a0

Fuster, J. M., \& Jervey, J. P. (1981). Inferotemporal neurons distinguish and retain behaviorally relevant features of visual stimuli. Science, 212(4497), 952-955. https://doi.org/10.1126/science.7233192

Glanzer, M., \& Cunitz, A. R. (1966). Two storage mechanisms in free recall. Journal of Verbal Learning and Verbal Behavior, 5(4), 351-360. https://doi.org/10.1016/S00225371(66)80044-0

Harrison, S. A., \& Tong, F. (2009). Decoding reveals the contents of visual working memory in early visual areas. Nature, 458(7238), 632-635. https://doi.org/10.1038/nature07832

James, W. (1890). Priciples of psychology (Vol 1). https://doi.org/10.1037/10538-000

Jeneson, A., \& Squire, L. R. (2012). Working memory, long-term memory, and medial temporal lobe function. Learning and Memory, 19, 15-25. https://doi.org/10.1101//m.024018.111.19

Karpicke, J. D., \& Roediger, H. L. (2006). Test-enhanced learning: Taking memory tests improves long-term retention. Psychological Science, 17(3), 249-255.

https://doi.org/10.1111/j.1467-9280.2006.01693.x

Kiyonaga, A., Dowd, E. W., \& Egner, T. (2017). Neural representation of working memory content is modulated by visual attentional demand. Journal of Cognitive Neuroscience, 29(12), 2011-2024. https://doi.org/10.1162/jocn_a_01174

Kiyonaga, A., Scimeca, J. M., Bliss, D. P., \& Whitney, D. (2017). Serial dependence across perception, attention, and memory. Trends in Cognitive Sciences, 21(7), 492-493. https://doi.org/10.1016/j.tics.2017.04.009

Lamprecht, R., \& Ledoux, J. (2004). Structural plasticity and memory. Nature Reviews Neuroscience, 5, 45-54. https://doi.org/10.1038/nrn1301 
LaRocque, J. J., Lewis-Peacock, J. A., \& Postle, B. R. (2014). Multiple neural states of representation in short-term memory? It's a matter of attention. Frontiers in Human Neuroscience, 8(January), 1-14. https://doi.org/10.3389/fnhum.2014.00005

LaRocque, J. J., Lewis-Peacock, J. a, Drysdale, A. T., Oberauer, K., \& Postle, B. R. (2013). Decoding attended information in short-term memory: an EEG study. Journal of Cognitive Neuroscience, 25(1), 127-142. https://doi.org/10.1162/jocn_a_00305

Larocque, J. J., Riggall, A. C., Emrich, S. M., \& Postle, B. R. (2017). Within-category decoding of information in different attentional states in short-term memory. Cerebral Cortex, 27(10), 4881-4890. https://doi.org/10.1093/cercor/bhw283

Leavitt, M. L., Mendoza-halliday, D., \& Martinez-trujillo, J. C. (2017). Sustained activity encoding working memories: Not fully distributed. Trends in Neurosciences, 40(6), 328-346. https://doi.org/10.1016/j.tins.2017.04.004

Lebedev, M. A., Messinger, A., Kralik, J. D., \& Wise, S. P. (2004). Representation of attended versus remembered locations in prefrontal cortex. PLoS Biology, 2(11). https://doi.org/10.1371/journal.pbio.0020365

Lewis-Peacock, J. A., Drysdale, A. T., Oberauer, K., \& Postle, B. R. (2012). Neural evidence for a distinction between short-term memory and the focus of attention. Journal of Cognitive Neuroscience, 24(1), 61-79. https://doi.org/10.1162/jocn_a_00140

Loaiza, V. M., Duperreault, K. A., Rhodes, M. G., \& McCabe, D. P. (2014). Long-term semantic representations moderate the effect of attentional refreshing on episodic memory. Psychonomic Bulletin and Review, 22(1), 274-280. https://doi.org/10.3758/s13423-0140673-7

Loaiza, V. M., \& McCabe, D. P. (2012). Temporal-contextual processing in working memory: Evidence from delayed cued recall and delayed free recall tests. Memory and Cognition, 40(2), 191-203. https://doi.org/10.3758/s13421-011-0148-2

Luck, S. J., \& Vogel, E. K. (1997). The capacity of visual working memory for features and conjunctions. Nature, 390(6657), 279-281. https://doi.org/10.1038/36846

Luck, S. J., \& Vogel, E. K. (2013). Visual working memory capacity: from psychophysics and neurobiology to individual differences. Trends in Cognitive Sciences, 17(8), 391-400. https://doi.org/10.1016/j.tics.2013.06.006

Lundqvist, M., Herman, P., \& Miller, E. K. (2018). Working Memory: Delay Activity, Yes! Persistent Activity? Maybe Not. The Journal of Neuroscience, 38(32), 7013-7019. https://doi.org/10.1523/JNEUROSCI.2485-17.2018

Luria, R., Balaban, H., Awh, E., \& Vogel, E. K. (2016). The contralateral delay activity as a neural measure of visual working memory. Neuroscience and Biobehavioral Reviews, 62, 100-108. https://doi.org/10.1016/j.neubiorev.2016.01.003

McCabe, D. P. (2008). The role of covert retrieval in working memory span tasks: Evidence from delayed recall tests. Journal of Memory and Language, 58(2), 480-494. https://doi.org/10.1016/j.jml.2007.04.004

McEIree, B. (2006). Accessing recent events. Psychology of Learning and Motivation Advances in Research and Theory, 46(06), 155-200. https://doi.org/10.1016/S00797421(06)46005-9 
Mendoza-Halliday, D., Torres, S., \& Martinez-Trujillo, J. C. (2014). Sharp emergence of featureselective sustained activity along the dorsal visual pathway. Nature Neuroscience, 17(9), 1255-1262. https://doi.org/10.1038/nn.3785

Miller, E. K., Lundqvist, M., \& Bastos, A. M. (2018). Working Memory 2.0. Neuron, 100(2), 463475. https://doi.org/10.1016/j.neuron.2018.09.023

Mogle, J. A., Lovett, B. J., Stawski, R. S., \& Sliwinski, M. J. (2008). What's so special about working memory? Psychological Science, 19(11), 1071-1077. https://doi.org/10.1111/j.1467-9280.2008.02202.x

Mongillo, G., Barak, O., \& Tsodyks, M. (2008). Synaptic theory of working memory. Science, 319(5869), 1543-1546. https://doi.org/10.1126/science.1150769

Murray, J. D., Bernacchia, A., Roy, N. A., Constantinidis, C., Romo, R., Wang, X.-J., \& Performed, N. A. R. (2017). Stable population coding for working memory coexists with heterogeneous neural dynamics in prefrontal cortex. Proceedings of the National Academy of Sciences, 114(2), 394-399. https://doi.org/10.1073/pnas.1619449114

Oberauer, K. (2002). Access to information in working memory: Exploring the focus of attention. Journal of Experimental Psychology: Learning, Memory, and Cognition, 28(3), 411-421. https://doi.org/10.1037//0278-7393.28.3.411

Oberauer, K., Awh, E., \& Sutterer, D. W. (2017). The role of long-term memory in a test of visual working memory: Proactive facilitation but no proactive interference. Journal of Experimental Psychology: Learning Memory and Cognition, 43(1), 1-22. https://doi.org/10.1037/xIm0000302

Oberauer, K., \& Hein, L. (2012). Attention to Information in Working Memory. Current Directions in Psychological Science, 21(3), 164-169. https://doi.org/10.1177/0963721412444727

Oztekin, I., Davachi, L., \& McEIree, B. (2010). Are representations in working memory distinct from representations in long-term memory? Neural evidence in support of a single store. Psychological Science, 21(8), 1123-1133. https://doi.org/10.1177/0956797610376651

Postle, B. R. (2015). The cognitive neuroscience of visual short-term memory. Current Opinion in Behavioral Sciences, 1, 40-46. https://doi.org/10.1016/j.cobeha.2014.08.004

Radvansky, G. A., \& Zacks, J. M. (2017). Event boundaries in memory and cognition. Current Opinion in Behavioral Sciences, 17, 133-140. https://doi.org/10.1016/j.cobeha.2017.08.006

Ranganath, C., \& Blumenfeld, R. S. (2005). Doubts about double dissociations between shortand long-term memory. Trends in Cognitive Sciences, 9(8), 374-380.

https://doi.org/10.1016/j.tics.2005.06.009

Rao, S. C., Rainer, G., \& Miller, E. K. (1997). Integration of what and where in the primate prefrontal cortex. Science, 276(5313), 821-824.

https://doi.org/10.1126/science.276.5313.821

Reinhart, R. M. G., McClenahan, L. J., \& Woodman, G. F. (2016). Attention's accelerator. Psychological Science. https://doi.org/10.1177/0956797616636416

Rhodes, S., \& Cowan, N. (2018). Attention in working memory: Attention is needed but it yearns to be free. Annals of the New York Academy of Sciences, 1424, 52-63.

https://doi.org/10.1111/nyas.13652 
Roediger, H. L., \& Butler, A. C. (2011). The critical role of retrieval practice in long-term retention. Trends in Cognitive Sciences, 15(1), 20-27.

https://doi.org/10.1016/j.tics.2010.09.003

Rose, N. S., Buchsbaum, B. R., \& Craik, F. I. M. (2014). Short-term retention of a single word relies on retrieval from long-term memory when both rehearsal and refreshing are disrupted. Memory \& Cognition, 42(5), 689-700. https://doi.org/10.3758/s13421-014-0398$x$

Rose, N. S., Craik, F. I. M., \& Buchsbaum, B. R. (2015). Levels of processing in working memory: Differential involvement of frontotemporal networks. Journal of Cognitive Neuroscience, 27(3), 522-532. https://doi.org/10.1162/jocn_a_00738

Rose, N. S., LaRoque, J. J., Rigall, A. C., Gosseries, O., Starrett, M. J., Meyering, E. E., \& Postle, B. R. (2016). Reactivaion of latent working memories with transcranial magnetic stimulation. Science, 22(4), 265-266. https://doi.org/10.1126/science.aah7011

Salazar, R. F., Dotson, N. M., Bressler, S. L., \& Gray, C. M. (2012). Content-specific frontoparietal synchronization during visual working memory. Science, 338, 1097-1100.

Scoville, W. B., \& Milner, B. (1957). Loss of recent memory after bilateral hippocampal lesions. Journal of Neurology, Neurosurgery, and Psychiatry, 20(1), 11-21. https://doi.org/10.1136/jnnp.20.1.11

Serences, J. T. (2016). Neural mechanisms of information storage in visual short-term memory. Vision Research, 128, 53-67. https://doi.org/10.1016/j.visres.2016.09.010

Serences, J. T., Ester, E. F., Vogel, E. K., \& Awh, E. (2009). Stimulus-specific delay activity in human primary visual cortex. Psychological Science, 20(2), 207-214. https://doi.org/10.1111/j.1467-9280.2009.02276.x

Shipstead, Z., Lindsey, D. R. B., Marshall, R. L., \& Engle, R. W. (2014). The mechanisms of working memory capacity: Primary memory, secondary memory, and attention control. Journal of Memory and Language, 72(1), 116-141. https://doi.org/10.1016/j.jml.2014.01.004

Souza, A. S., \& Oberauer, K. (2017). Time to process information in working memory improves episodic memory. Journal of Memory and Language, 96, 155-167. https://doi.org/10.1016/j.jml.2017.07.002

Sprague, T. C., Ester, E. F., \& Serences, J. T. (2014). Reconstructions of information in visual spatial working memory degrade with memory load. Current Biology, 24(18), 1-7. https://doi.org/10.1016/j.cub.2014.07.066

Sprague, T. C., Ester, E. F., \& Serences, J. T. (2016). Restoring latent visual working memory representations in human cortex. Neuron, 91(3), 694-707. https://doi.org/10.1016/j.neuron.2016.07.006

Sreenivasan, K. K., Curtis, C. E., \& D’Esposito, M. (2014). Revisiting the role of persistent neural activity during working memory. Trends in Cognitive Sciences, 18(2), 82-89. https://doi.org/10.1016/j.tics.2013.12.001

Sreenivasan, K. K., \& D'Esposito, M. (2019). The what, where and how of delay activity. Nature Reviews Neuroscience, 10-14. https://doi.org/10.1038/s41583-019-0176-7

Stokes, M. G. (2015). 'Activity-silent' working memory in prefrontal cortex: a dynamic coding 
framework. Trends in Cognitive Sciences, 19(7), 394-405.

https://doi.org/10.1016/j.tics.2015.05.004

Stokes, M. G., Kusunoki, M., Sigala, N., Nili, H., Gaffan, D., \& Duncan, J. (2013). Dynamic coding for cognitive control in prefrontal cortex. Neuron, 78(2), 364-375. https://doi.org/10.1016/j.neuron.2013.01.039

Sutterer, D. W., Foster, J. J., Adam, K. C. S., Vogel, E. K., \& Awh, E. (2019). Item-specific delay activity demonstrates concurrent storage of multiple items in working memory. PLoS Biology, 17(4), e3000239. https://doi.org/10.1101/382879

Todd, J. J., \& Marois, R. (2004). Capacity limit of visual short-term memory in human posterior parietal cortex. Nature, 428(6984), 751-754. https://doi.org/10.1038/nature02466

Todd, J. J., \& Marois, R. (2005). Posterior parietal cortex activity predicts individual differences in visual short-term memory capacity. Cognitive, Affective \& Behavioral Neuroscience, 5(2), 144-155. https://doi.org/10.3758/CABN.5.2.144

Turner, M. L., \& Engle, R. W. (1989). Is working memory capacity task dependent? Journal of Memory and Language, 28(2), 127-154. https://doi.org/10.1016/0749-596X(89)90040-5

Underwood, B. J. (1957). Intereference and forgetting. Psychological Review, 64(1), 49-60. https://doi.org/10.1037/h0044616

Unsworth, N., Brewer, G. A., \& Spillers, G. J. (2009). There's more to the working memory capacity-fluid intelligence relationship than just secondary memory. Psychonomic Bulletin and Review, 16(5), 931-937. https://doi.org/10.3758/PBR.16.5.931

Unsworth, N., \& Engle, R. W. (2006). Simple and complex memory spans and their relation to fluid abilities: Evidence from list-length effects. Journal of Memory and Language, 54(1), 68-80. https://doi.org/10.1016/j.jml.2005.06.003

Unsworth, N., \& Engle, R. W. (2007). The nature of individual differences in working memory capacity: Active maintenance in primary memory and controlled search from secondary memory. Psychological Review, 114(1), 104-132. https://doi.org/10.1037/0033295X.114.1.104

Unsworth, N., Fukuda, K., Awh, E., \& Vogel, E. K. (2014). Working memory and fluid intelligence: Capacity, attention control, and secondary memory retrieval. Cognitive Psychology, 71, 1-26. https://doi.org/10.1016/j.cogpsych.2014.01.003

Unsworth, N., Fukuda, K., Awh, E., \& Vogel, E. K. (2015). Working memory delay activity predicts individual differences in cognitive abilities. Journal of Cognitive Neuroscience, 27(5), 853-865. https://doi.org/10.1162/jocn_a_00765

Unsworth, N., \& Spillers, G. J. (2010). Working memory capacity: Attention control, secondary memory, or both? A direct test of the dual-component model. Journal of Memory and Language, 62(4), 392-406. https://doi.org/10.1016/j.jml.2010.02.001

Unsworth, N., Spillers, G. J., \& Brewer, G. A. (2010). The contributions of primary and secondary memory to working memory capacity: An individual differences analysis of immediate free recall. Journal of Experimental Psychology: Learning Memory and Cognition, 36(1), 240-247. https://doi.org/10.1037/a0017739

van Moorselaar, D., Foster, J. J., Sutterer, D. W., Theeuwes, J., Olivers, C. N. L., \& Awh, E. (2018). Spatially selective alpha oscillations reveal moment-by-moment trade-offs between 
working memory and attention. Journal of Cognitive Neuroscience, 30(2), 256-266. https://doi.org/10.1162/jocn_a_01198

Vogel, E. K., \& Machizawa, M. G. (2004). Neural activity predicts individual differences in visual working memory capacity. Nature, 428(6984), 748-751. https://doi.org/10.1038/nature02447

Warrington, E. K., \& Shallice, T. (1969). The selective impairment of auditory verbal short-term memory. Brain, 92, 885-896. https://doi.org/10.1093/brain/92.4.885

Watanabe, K., \& Funahashi, S. (2014). Neural mechanisms of dual-task interference and cognitive capacity limitation in the prefrontal cortex. Nature Neuroscience, 17(4), 601-611. https://doi.org/10.1038/nn.3667

Waugh, N. C., \& Norman, D. A. (1965). Primary memory. Psychological Review, 72(2), 89-104. https://doi.org/10.1001/archderm.1976.01630340034009

Williams, M., \& Woodman, G. F. (2012). Directed forgetting and directed remembering in visual working memory. Journal of Experimental Psychology. Learning, Memory, and Cognition, 38(5), 1206-1220. https://doi.org/10.1037/a0027389

Wolff, M. J., Ding, J., Myers, N. E., \& Stokes, M. G. (2015). Revealing hidden states in visual memory using electroencephalography. Frontiers in Systems Neuroscience, 9(September), 1-12. https://doi.org/10.3389/fnsys.2015.00123

Wolff, M. J., Jochim, J., Akyurek, E. G., \& Stokes, M. G. (2017). Dynamic hidden states underlying working-memory-guided behaviour. Nature Neuroscience. https://doi.org/10.1038/nn.4546

Yu, Q., \& Shim, W. M. (2017). Occipital, parietal, and frontal cortices selectively maintain taskrelevant features of multi-feature objects in visual working memory. Neurolmage, 157, 97107. https://doi.org/10.1016/j.neuroimage.2017.05.055

Zhang, W., \& Luck, S. J. (2008). Discrete fixed-resolution representations in visual working memory. Nature, 453(7192), 233-235. https://doi.org/10.1038/nature06860 